\title{
La máquina musical en "El perseguidor" de Julio Cortázar
}

And rés González Riquelme

Universidad de Concepción

\section{Resumen}

El propósito de este artículo es estudiar el influjo del jazz en la producción del relato “El perseguidor" de Julio Cortázar. Para este efecto, se ha trabajado con el marco teórico de M il mesetas, de D eleuze y G uattari, y con la terminología específica con que se describe el jazz.

Palabras claves: "El perseguidor", literatura y jazz, influjo.

\section{ABST RACT}

The purpose of this article is to study the flowin of jazz music in the production of Julio Cortazar's story "El perseguidor". In order to do this, it has been worked with thetheoretical framework of $\mathrm{M}$ il mesetas, by Deleuze and Guattari and with the specific terminology used to describe jazz.

Keywords: "El perseguidor", literature and jazz, influx.

Recibido: 13.11.2002. Aceptado: 19.11.2002.

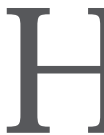

AY UNA RELACION explícita y evidente entre el relato "EI perseguidor", de Julio Cortázar, y el jazz, la que ya ha sido descrita en otros estudios y que cualquier conocedor de la historia del jazz y, específicamente, de la vida y circunstancias de C harlie Parker, advierte con facilidad. Lo que no resulta tan evidente es que este relato - su escritura, sus personajes, su problemática- está bajo un fuerte influjo del jazz. Pero esta afirmación puede resultar vaga si no se precisan las relaciones específicas entre el jazz y el relato en cuestión. M ás aún, podemos enunciar que este relato está maquinado por el jazz, está completamente atravesado por la máquina musical jazzística. C reemos quela concepción del texto, quesu escritura, están bajo un influjo específico del jazz que acaso es posible precisar de la misma manera en que se describe el 
influjo de un autor o un estilo sobre otro. Lo importante es establecer una relación entre "El perseguidor" y el jazz que vaya más allá del vínculo explícito y evidente. Para esto, entonces, habría que ver cuáles son los elementos de la máquina musical jazzística y cómo funciona. Y luego, de qué manera este mismo funcionamiento opera en el relato y participa de su producción, o lo que es lo mismo, de qué manera este relato es maquinado por el jazz. U sualmente, los elementos con que se describe el funcionamiento de un arte no se aplican a otro, sino que permanecen celosamente guardados en su dominio específico, donde han encontrado su origen y adquieren su precisión. Pero a nosotros nos inquieta cómo es que la literatura pueda estar bajo el influjo de la música, lo cual no es lo mismo que decir que aquella obra literaria fue inspirada por aqueIla obra musical. Lo interesante es precisar ese influjo, trazar esas relaciones, verificar qué cosa específica y singular de un arte ha influido sobre otra. El problema es cómo, a partir de esas relaciones, se pueda componer una máquina que permita otras múltiples conexiones, y que saque a los elementos específicos que constituyen cada dominio del aislamiento en que permanecen. $Y$ en nuestro caso, verificar qué hay de específico y singular en el jazz que desencadenó la producción de todo un relato. N uestra especialidad no es la musicología, pero guiados por un excelente teórico de jazz, Joachim Berendt, hemos entrado en ese dominio y hemos tomado de allí algunos conceptos que definen elementos específicos del jazz: off-beat, swing, bebop, quinta di sminuida o bluenote, con los cuales podremos describir el funcionamiento básico de lo que hemos llamado máquina musical jazzística.

C omo se podrá advertir, hemos hablado en términos de máquina musical y no en términos de estilo o tendencia musical. El concepto de máquina musical tiene una particular precisión y belleza teóricas que nos abre la posibilidad de describir con mayor felicidad el fenómeno que nos inquieta. Asimismo, para poder trazar algunas líneas, para poder realizar al gunas conexiones, se nos hace necesario utilizar al gunos otros conceptos de D eleuze y G uattari, mas no para adscribirnos a una moda extranjerizante ni participar en polémicas en curso, sino por el simple hecho de que nos resultan funcionales dada su calidad de abstracción y las múltiples relaciones que permiten establecer. Así, hemos iniciado esta investigación sobre la base de los siguientes enunciados: 1) las líneas defuga se relacionan unas con otras, se tocan, se alteran; 2) una línea de fuga en un dominio específico (por ejemplo, la música) no es ajena a líneas de fuga en otros dominios diferentes (por ejemplo, la literatura); las líneas de fuga pueden desencadenarse por contagio: el influjo de un dominio en otro muy diferente.

Lo que nos interesa, más que interpretar o explicar un objeto de estudio determinado, es establecer conexiones, conectar la obra literaria con elementos de dominios diferentes. Referirse, más que a la obra en sí, a los elementos con 
que se conecta. C reemos que a partir de esta operación, se abrirá la compresión de la obra. Abrirse: permitir el paso de otras cosas, salir al encuentro. N o descifrar ni explicar nada. La obra no necesita ser esclarecida: es clara en sí misma, se explica a sí misma sin la necesidad de ninguna terminología superior a la cual se someta. Lo que nos inquieta es llevar los problemas que se tratan en la obra a otros planos, no superiores, sino solamente distintos, y con los cuales se relaciona. La operación consiste, entonces, en transportarla. La necesidad de emplear otra terminología, tal vez más abstracta, no es para descifrar, sino para hacer múltiples conexiones. Por lo tanto, lo que requiere de explicación no es la obra en sí misma, no son los problemas que allí se tratan, sino las líneas de conexión. Son ésas las que nos resultan interesantes describir y explicar: ¿en quéy cómo se relaciona específicamente el relato con el jazz?

II

\section{LA MAQUINA MUSICAL Y EL JAZZ}

Lo que decimos, entonces, es que en el relato hay la participación de una máquina musical, de una máquina musical que guarda relaciones específicas con el jazz, o que, dicho de otro modo, cuya relación con el jazz no es casual. No se trata de que Cortázar haya hecho música. Tampoco se propone hablar específica y únicamente del jazz. Lo que él hace es componer un relato en el que entra en relación con una máquina musical, donde se produce un agenciamiento entre dos dominios diferentes: música y literatura. El relato está basado en la vida de un músico, Charlie Parker, con el que guarda evidentes relaciones biográficas (C fr. Arellano, 1972: 64-67). Pero eso no estodo, es sólo lo más evidente. Digamos que en el relato, al tratarse acerca de las extrañas relaciones que un músico de jazz tiene con el tiempo y la realidad, y del conflicto entre éstey un crítico de jazz; 0 al tratarse acerca de un crítico de jazz que se ve envuelto por un músico de jazz que le fascina (da lo mismo cómo se enuncie), se produce una conexión con elementos específicos de la música de jazz, con elementos propios de la estructura del jazz, con lo que en el jazz hay de huidizo y rupturista, con lo que es inmanente a esta música y que está entre sus cual idades más relevantes. ¿Porqué sino Julio Cortázar ha elegido a un músico de jazz para hacer un relato donde se problematiza el tiempo y la libertad, la creación o búsqueda de espacios de libertad?

El relato "El perseguidor" está completamente atravesado por la máquina musical. ¿Q ué quiere decir esto? Entendemos aquí máquina en el sentido de un encuentro y conexión entre distintos elementos, los que se agencian para fun- 
cionar conjuntamenteen un movimiento quelos arrastra a todos. Setrata de un co-funcionamiento entre distintos elementos. U na máquina de este tipo es una máquina que se compone en función del movimiento, que produce movilizaciones de fuerzas. Sólo movimientos y variaciones continuas. Fugas en múltiples direcciones. Por eso, se trata de una máquina de guerra nómada: lo que importa es el avanzar, no interesa hacia dónde, pues nunca hay un fin determinado, sólo el espacio abierto de las movilidades y de la variación continua. Si esta máquina de guerra, en su continuo avanzar, pasa por la música, la arrastra a la composición de una máquina concreta llamada máquina musical. Lo mismo cuando atraviesa la literatura: una máquina literaria. Arrastra elementos a su paso, los aglomera, los relaciona, los conecta, los hace co-funcionar. Eso es componerlos. Pero todo esto es sólo una manera de decirlo; también se puede de otra forma. Se puede decir que son los flujos los que, impelidos por el puro movimiento y las fuerzas del devenir, componen una máquina de guerra con la que se asegura o busca la movilidad continua. Y estos movimientos lo atraviesan... 0 intentan atravesarlo todo. Cuando esos movimientos pasan por la literatura (o cuando la literatura tiende hacia ellos o se encuentra con ellos), se compone un tipo concreto de máquina de guerra a la que se le llama máquina literaria: una producción continua de variaciones a nivel del lenguaje, de las palabras, de la escritura. Lo mismo cuando pasa o se encuentra con la música. Y también se puede volver a decir todo de otra manera. Por ejemplo, tenemos una máquina musical, y produce variaciones continuas que se expresan en el ámbito de los tonos y los ritmos. Y esas variaciones continuas, en ese espacio -en el de la música- desencadenan otras movilizaciones, arrastra otros elementos a su paso. Se agencia con otras cosas, siempreen función del movimiento y del devenir, delas variaciones continuas. Así, de pronto, se encuentra con la literatura, y se agencia con ella. De ese encuentro surge algo así como el relato con el que estamos trabajando. Por eso hemos dicho que en este relato hay la participación de una máquina musical, una máquina musical específica relacionada con el jazz.

El mismo Julio Cortázar, como escritor, es arrastrado por esas variaciones continuas que se dan en la música y bajo ese influjo, digamos, compone un relato quees, entonces, inseparable del jazz. Los movimientos de desterritorialización que se expresan a través de la música lo tocan a él, lo maquinan para crear un relato donde esos mismos movimientos se expresan a través de personajes. Para ser más convincentes, hemos elegido un relato en el que los personajes se relacionan explícita y evidentemente con la música: músicos de jazz, amigos de músicos y un crítico de jazz. La música los atraviesa a todos ellos, los descoloca, los hace moverse de diversas maneras. Por ejemplo, la marquesa Tica: "Cuando la marquesa echa a hablar uno se pregunta si el estilo de Dizzy no se le ha pegado al idioma, pues es una serie interminable de variaciones en los registros 
más inesperados, hasta que al final la marquesa se da un gran golpe en los muslos, abre de par en par la boca y se pone a rér como si la estuvieran matando a cosquillas" (C ortázar, 1980: 307). D espués de esta cita, nos sentimos tentados a decir quela marquesa T ica está henchida de bebop. El relato en su totalidad está henchido de bebop, y la problemática del tiempo tan insistentemente expuesta a lo largo del mismo, no esajena al jazz y a la manera en que esta música despliega su temporalidad, expresando una tensión rítmica que desborda la música y toca a los oyentes, Ilena los espacios y altera la habitual percepción uniforme de las cosas.

Ahora bien, ¿qué relación puede haber entre una alteración perceptual del tiempo, tal como se expresa en el relato, y la música, y específicamente en este relato, con el jazz? ¿Q ué hay, entonces, en el jazz, que lo conecta con estos problemas?

En efecto, el problema del tiempo es el problema de la música, y "el problema de la música es el de una potencia de desterritorialización que atraviesa a la natural eza, los animales, los elementos y los desiertos no menos que el hombre" (D eleuze y Guattari, 1997: 307). En el caso del relato que nos interesa, se trata de una desterritorialización del tiempo, del flujo-tiempo, potenciada a través de la música que ejecuta un músico de jazz. Toda esta música, todo el jazz está atravesado por series continuas de fugas de los tiempos estables y de los acentos (beat), para producir líneas de improvisación dinámicas eirregulares que permiten la proliferación y el enriquecimiento de las líneas melódicas:

... forma parte del swing la pluralidad de ritmos y la tensión entre ellos; es decir, el desplazamiento de los acentos rítmicos (...) Este desplazamiento se llama en la música europea śncopa. En la música europea síncopa significa un desplazamiento exactamente definido del centro de gravedad del compás; el acento cae precisamente en la mitad dela distancia entre dos golpes. En el jazz, por el contrario, las desviaciones del acento son más libres, flexibles y sutiles: el acento puede caer en cualquier lugar de la distancia entre dos beats (...) C omo ese acento se mueve alejándose del beat -y sin embargo lo destaca en la negación- se le llama off-beat (Berendt, 1998: 304).

Este es uno de los aspectos más característicos del jazz, y lo que, entre otros elementos, tiene de revolucionario en la música. Este elemento rítmico, el offbeat, asegura una constante fuga del tiempo pul sado (beat), para abrir posibilidades de improvisación con múltiples huidas. Junto con otros elementos, el offbeat permite abrir una línea melódica para introducir en ella muchas variantes, que es lo que suelen hacer los músicos de jazz al interpretar una y otra vez los mismos temas standards: introducir variantes a la línea original de una pieza musical determinada. Al introducir estas variantes, ino está un músico haciendo 
huir esa pieza musical sobre la cual trabaja? Consideremos una pieza musical (una canción, un tema) como un sistema de coordenadas fijas y estables. Esa pieza, ese sistema de coordenadas, en el caso del estilo bebop "es presentado al unísono al comienzo y al final de cada pieza, tocado generalmente por dos instrumentos de viento, las más de las veces una trompeta y un saxofón" (Berendt, 1998: 45). La improvisación, o lo que se llama chorus phrase, se realiza en el medio. El tema es sólo la base de la cual el músico extrae material para producir chorus phrase. Yendo aún más lejos, Berendt sostiene que "el tema sobre el que se improvisa se ha hecho cada vez menos importante en el curso de la evolución del jazz (...) se improvisa con tanta libertad que la melodía carece ya de importancia. Con frecuencia no puede reconocerse ni en los comienzos de las piezas" (Berendt, 1998: 323). Y añadiendo otra cita del musicólogo, variamos lo que un poco más arriba habíamos afirmado: "El músico de jazz no improvisa sobre un tema, sino sobre armonías..." (Berendt, 1998: 323). Por eso utilizaremos la noción desistema decoordenadas, pues nos parece mucho más conveniente, dada su abstracción y la posibilidad que nos abre para hacer las conexiones que nos importan.

Todo el jazz, toda su historia, es un constante abrir posibilidades para líneas de improvisación cada vez más libres. Allí donde de pronto se produce una obstrucción de los flujos, allí donde la música se estanca en formas anquilosadas, aparecen músicos revolucionarios que aseguran la fuga de la música y la apertura de nuevas y múltiples posibilidades de continuidad y proliferación. Es así como Bruno se refiere a Johnny Carter: "Johnny ha pasado por el jazz como una mano que da vuelta la hoja, y se acabó" (C ortázar, 1980: 305). Aquí nos conectamos con otro aspecto importante del jazz, a saber, con su historia, y con uno de los estilos que revolucionó drásticamente el jazz en la década de los cuarenta: el bebop.

La palabra bebop "refleja onomatopéyicamente el intervalo más popular de la época: la quinta disminuida descendente. Las palabras bebop o rebop se formaban por sí solas cuando se querían cantar tales saltos melódicos" (Berendt, 1998: 43). El empleo de la quinta disminuida es fundamental en el bebop. Se trata de un intervalo que antes se percibía como equivocado o disonante. Un intervalo cuya sonoridad escapa a las normas tradicionales de la armonía.

Este intervalo, la quinta disminuida, constituye también lo que se conoce como una blue note, que es otro elemento característico y fundamental en el jazz, y que importa a la armonía. Se trata de otro rasgo que pone en variación la armonía tradicional y que produce alteraciones armónicas que no se pueden situar en un punto fijo: "Las bluenotes no setratan en lo absoluto de disminuciones de un semitono, sino disminuciones microtonales mucho más pequeñas" (Berendt, 1998: 287). Existen, por un lado, las notas fijas y estables. La emergencia de blue notes en una línea melódica altera de tal modo la secuencia de 
notas de esa línea, que le permite huir de las fijaciones sonoras a las que están sujetas las notas. 0 tra vez, huida de las unidades estables.

El improvisador se mueve, entonces, entre esas coordenadas fijas y estables, pero para mantener tensión con ellas, para extraer de allí variaciones continuas. Es importante aquí el valor de las palabras entre o medio tal como las utilizan y trabajan D eleuze y G uattari. Los movimientos de fuga y de desterritorialización siempre se producen entre los puntos fijos y estables, que constituyen territorios. En una línea de fuga, en un camino, lo que importa "nunca es el principio ni el final, siempre es el medio" (D eleuze y Parnet, 1980: 34). No se trata de situarse en un territorio determinado, sino pasar entre. $Y$ la manera como un músico de jazz pasa entre, o la manera como produce variaciones continuas, consiste básicamente en desplazamientos rítmicos y microtonales, que permiten a su vez el desprendimiento de nuevas líneas melódicas, aceleraciones 0 lentitudes, llevando la ejecución musical a un máximo de tensión entre los elementos que la constituyen. Y era tanta la tensión que, por ejemplo, un músico como Charlie Parker provocaba con sus improvisaciones, que M iles D avis ha Ilamado a esto "poner de cabeza la sección rítmica". Y acerca de las sesiones con Charlie Parker, añade: "Estábamos tocando por ejemplo un blues cuando de repente Bird (Charlie Parker) comenzaba a improvisar en el compás once, y como la sección rítmica se quedaba donde estaba y Bird seguía tocando por su camino, sonaba como si el ritmo se acentuara en el uno y en el tres en lugar del dos y el cuatro. Siempre que ocurría esto le gritaba $M$ ax Roach, el baterista, a D ukeJ ordan, el pianista, que no siguiera a Bird, sino quesiguiera en lo que ellos estaban tocando. Con esto se produjo finalmente lo que Bird deseaba y nos juntamos otra vez todos" (Berendt, 1998: 336-337). Así un improvisador de jazz pasa a través de las unidades estables de una pieza estándar, y abre una y otra vez espacios entre, líneas flotantes, off-beat.

D e esta manera, entonces, el jazz pone en movimiento, hace pasar flujos desterritorializados: flujos sonoros, rítmicos y temporales. D eleuze: "La música emite flujos moleculares" (1997: 307). Flujos que escapan a lo establecido, a lo reconocible, situable y clasificable. C ortázar: "Este jazz desecha todo erotismo fácil... para situarse en un plano aparentemente desasido donde la música queda en absoluta libertad (...) una música que no facilita los orgasmos ni las nostalgias" (1980: 313).

Tenemos, en definitiva, los siguientes elementos, o dicho de otra manera, las siguientes piezas de la máquina musical: off-beat, blue note, y quinta disminuida en el caso del bebop. Todas ellas, tal como las hemos definido, suponen líneas de fuga o movimientos de desterritorialización. Fuga de los puntos rítmicos: off-beat. Fuga de los puntos armónicos: blue note y quinta disminuida. Variaciones microtonales y desviaciones sutiles del acento rítmico. Todos estos movi- 
mientos nos remiten a la noción de entre o de medio. Son estos espacios, desterritorializados, los que permiten las exploraciones creativas, las líneas de fuga que hacen huir los sistemas para hacer pasar los flujos (flujos rítmicos, melódicos, armónicos, sonoros: moleculares), asegurando así la variación continua, el paso y desarrollo de las multiplicidades. Cortázar: "Una construcción infinita cuyo placer no está en el rematesino en la reiteración exploradora, en el ejemplo de facultades que dejan atrás lo prontamente humano sin perder humanidad" (1980: 313).

III

\section{LA MAQUINA MUSICAL Y EL PROBLEMA DEL TIEM PO: CRONOS / AIÔN}

En el relato "El perseguidor" hay una problemática del tiempo que consiste básicamente en la oposición entre dos temporalidades. Una, la normal, la habitual, la que es mensurable en las medidas estables del reloj. La otra, una temporalidad que se manifiesta a través de Johnny Carter, y que no se deja medir ni atrapar por las medidas estables. A hora bien, ¿cómo tratar este problema, esta oposición entre temporalidades? ¿Cómo definirlo o describirlo, cómo hacerlo funcional dentro de una descripción teórica? ¿Y cómo relacionarlo, finalmente, con el jazz? Nos parece que este conflicto de temporalidades no es ninguna vaguedad ni capricho de Julio Cortázar, no es ninguna arbitrariedad de la imaginación, no es nada que no se pueda conectar con otra cosa y que no se pueda describir (medianamente, por supuesto) o relacionar con otros términos. Si el problema se propone con tanta insistencia en el relato, es porque guarda al guna relación esencial con otras cosas que van más allá del mismo relato. Por ejemplo, nos hemos referido a la música de jazz, y de qué menara en esta música se producen desterritorializaciones, fugas de las unidades estables que la rigen, alteraciones rítmicas y armónicas. Y lo rítmico está intrínsecamente relacionado con lo temporal. Ritmo es la "proporción guardada entreel tiempo de un movimiento y el de otro diferente" (Brenet, 1962: 457). El ritmo es toda una relación específica entre movimientos, entre las temporalidades de movimientos diferentes. Entonces vemos que este problema de la temporalidad no importa sólo al relato en cuestión, sino también a la música en su totalidad. Y en el relato hay una explícita relación entre el problema del tiempo y la música: "Yo creo que la música ayuda siempre a comprender un poco este asunto (...) la música me metía en el tiempo" (C ortázar, 1980: 293-295). ¿Cómo conectar una cosa con otra? Para esclarecer más el problema y establecer relaciones, recurriremosa 
otras nociones trabajadas por D eleuze y Guattari: Cronosy Aiôn. Así, al primer tiempo, al tiempo normal y mensurable en las medidas estables, lo llamaremos Cronos; a la otra temporalidad, la llamaremos Aiôn.

Cronos es, entonces, el tiempo de las medidas estables, el tiempo cronomé trico y normal, profundamente implicado con las normas sociales, un tiempo que "fija las cosas y las personas, desarrolla una forma y determina un sujeto" (D eleuzey G uattari, 1997: 265). Aiôn es el tiempo desterritorializado: "El tiempo indefinido del acontecimiento, la línea flotante que sólo conoce las velocidades, y que no deja de dividir lo que ocurre en un déjà-la y un pasencorelà, un demasiado tarde y un demasiado pronto simultáneos, un al go que sucederá y que a la vez acaba de suceder" (D eleuze y G uattari, 1997: 265). Esta definición se acopla, se conecta perfectamente con las explicaciones del mismo Johnny Carter: "Esto lo estoy tocando mañana... esto ya lo toqué mañana..." (C ortázar, 1980: 292). Es decir, acaba de suceder y a la vez sucederá. Toda una alteración de las habituales coordenadas de espacio-tiempo que saca extrañas fórmulas de lenguaje para expresarse, que hace decir a Johnny oraciones que no se deben decir, oraciones absurdas, pero que son la única manera de poder dar cuenta de esas experiencias que lo al teran y lo arrastran continuamente.

A caba de suceder y a la vez sucederá: ¿Cómo es eso posible? ¿Q uées eso, "un al go que sucederá y que a la vez acaba de suceder"? Esto tal vez se haga un poco más concreto al relacionarlo con la música. Y ésta no es una operación arbitraria de nuestra parte, pues en la misma definición que Deleuze y Guattari dan a propósito de C ronos y Aiôn, se establece una conexión con la música. Así, refiriéndose ellos a Pierre Boulez, afirman: "Boulez distingue en la música el tempo y el no tempo, el tiempo pulsado de una música formal y funcional basada en los valores, y el tiempo no pulsado para una música flotante, flotante y maquínica, que sólo tiene velocidades o diferencias de dinámica" (D eleuzey G uattari, 1997: 265). Relacionando los términos de la cita anterior, tempo y no tempo, con los que hemos definido a propósito del jazz, podemos establecer que beat (golpe 0 pulso) se relaciona con tempo, en tanto que off-beat con no tempo. Se trata de una diferencia terminológica, pues en el jazz se emplean algunos términos propios para esta música, que en la música europea estudiada por Boulez no se utilizan. Pero si relacionamos las definiciones, tenemos que setrata básicamente de lo mismo, sin el temor de ser demasiado arbitrarios. Veamos. En la teoría del jazz se habla de beat, y éste se define como: "un ritmo fundamental único marcado uniformemente durante toda la pieza: es el pulso de una obra de jazz (...) Este ritmo fundamental constituye el factor de orden: según él se ordena el fenómeno musical. Lo proporciona el baterista, aunque en el jazz moderno está también con frecuencia a cargo de los cuatro cuartos uniformes del bajista. Esta función ordenadora responde a una necesidad europea" (Berendt, 1998: 331). 
El beat es, entonces, el golpe, una marca uniforme fundamental: el tiempo pulsado. Pero el jazz tiene eso que llaman swing (no el estilo Swing de las grandes orquestas), aquello tan singular que lo distingue de la música europea. Y el swing surge, entre otros elementos menos definidos, por la tensión que se produce entre el beat y el off-beat. Y el off-beat ha sido definido como una desviación sutil y libre del beat: el "tiempo no pulsado de una música flotante...". Son importantes aquí al gunas palabras. Por ejemplo, flotante, una música flotante. Eso lo dicen D eleuze y Guattari, y la relación con Aiôn también la establecen ellos. En el jazz se habla de swing, del elemento rítmico swing. Y el problema del swing tiene mucho de "flotante", incluso si nos remitimosa la raíz inglesa de la pal abra. Ahora bien, hemos afirmado que el swing se da por una tensión entre beat y off-beat. Pero eso no es todo. El asunto es mucho más complejo. Se trata del encuentro entre temporalidades diferentes, que entran en conflicto y se resuelven, una y otra vez, y todo al mismo tiempo, no una cosa después de la otra, no una negación y después una afirmación, sino una tensión continua einstantánea entre una pluralidad de ritmos. N osotros nos hemos referido principalmente al off-beat porque es un término relativamente definido y práctico. Pero el mismo Berendt afirma que los off-beats "no generan por fuerza swing" (Berendt, 1998: 341). El problema del swing estriba en lo indefinible que tiene, aquello "flotante" que hay en él y quesin embargo se puede percibir al escuchar jazz. Lo que nos importa acerca del swing, es el hecho de que en él se efectúen constantes movimientos de fuga de las unidades estables, expresadas o manifestadas por esa tensión continua que se produce entre las distintas temporalidades.

El problema del jazz, del swing, es, sin lugar a dudas, el del tiempo. Berendt: "¿A quétiempo se refiereel swing?" (1998: 343). Esa misma preguntala podríamos poner en boca de Johnny $C$ arter cuando se deshace en alucinantes explicaciones acerca de susfugas del tiempo y sus entradas en otras temporalidades: "... la música me sacaba del tiempo... la música me metía en el tiempo" (Cortázar, 1980: 295). Y el tiempo al quese refiere cuando dice que entra en él impelido 0 arrastrado por la música, es ese tiempo flotante que no se deja mensurar, que no se deja atrapar por las medidas estables. Aquella temporalidad queal aludirla, al querer referirse a ella, al querer asirla de alguna manera, hace decir oraciones absurdas: "... esto ya lo toqué mañana" (Cortázar, 1980: 292). Y todo esto, como ya lo hemos señalado, se conecta con las definiciones dadas por D el euzey Guattari a propósito de Aiôn. Un "tiempo indefinido" - añadiríamos, además, rebosante de swing-, que no deja de emitir partículas de tiempo desterritorial izadas cuyo contacto hace variar la percepción habitual del tiempo, la vivencia del tiempo. No en vano esas sensaciones Johnny Carter las experimenta sobre todo cuando improvisa su música: "... esa improvisación, Ilena de huidas en todas direcciones" (Cortázar, 1980: 324). 
¿N o nos proporciona el arte, entre otras cosas, maneras diferentes, no habituales, de percibir y vivir la realidad, conexiones con otras cosas, planos de encuentros, salidas y entradas? ¿N o debería, en este caso la música, poner en variación y movimiento nuestra habitual percepción de las cosas, o nuestra habitual participación con ellas? Y si la música está intrínsecamente relacionada con el tiempo, ¿no nos debehacer variar la percepción y vivencia del mismo, permitirnos la proyección o creación de otros espacios y otras temporalidades? ¿Q ué sentido tendría redundar en lo mismo, en lo habitual y mensurable hasta el hartazgo? Esos son los problemas que están en juego en el relato, y con los cuales se relaciona la máquina musical en tanto que máquina de guerra. La máquina musical agenciada con la máquina literaria, a partir de cuyo encuentro se crea este relato: su lenguaje, sus personajes, sus acciones y conflictos, sus desesperaciones. Una máquina de guerra nómada que no deja de producir movilizaciones de fuerzas, movilizaciones deflujos, y en estecaso específico, la movilización del flujo-tiempo. Pues, en efecto, lo que aquí está en juego son movimientos de flujos: flujos desterritorializados que generan conflictos con el orden establecido. Si nos hemos referido bastanteal jazz, lo hemos hecho por lo siguiente: si las vivencias que Johnny Carter padece están intrínsecamente relacionadas con la música de jazz, ¿qué hay en él que produce todos estos movimientos, toda esta emisión de partículas de tiempo desterritorializadas? Así, hemos establecido las siguientes conexiones, que nos han servido para describir la máquina musical: off-beat - swing - blue note- bebop. Y a su vez, hemos relacionado esta máquina con el Tiempo de Carter - Aiôn.

Pero no queremos establecer, como tal vez se haya comprendido, una relación causal entre el jazz y las alteraciones temporales. Tal vez la causalidad esté implicada. No obstante, aunque esto ya no suene tan satisfactorio desdeel punto de vista de la razón, lo que se produce es una maquinación completa einstantánea entre la música y el tiempo. La música produce temporalidad. N o sólo se despliega en el tiempo y el espacio. Es ella misma una forma detemporalidad. Y su temporalidad, en el caso del jazz, es múltiple, según cómo se ha descrito el funcionamiento y el procedimiento de desterritorialización temporal queseejecuta. Tal vez sea la música la que, en su despliegue, produzca estas alteraciones de temporalidad. Tal vez sean otras sutilezas de percepción del tiempo las que produzcan esta música. Acaso sea una dinámica constante entre ambas cosas, y estemos siempre en el entrecruzamiento de diferentes temporalidades, una de las cuales (Cronos) tenga la pésima costumbre de tomarse el espacio completo de nuestras vivencias, mientras las otras (las Aiôn) fluyan desapercibidas en algunas músicas, se reclamen en algunos relatos. Si invocamos otras obras de Cortázar (Rayuela, "El otro cielo", "Ahí pero dónde, cómo") tenemos que siempre se alude a temporalidades-otras, fugas del tiempo, agujeros, "el otro lado", 
"el lado de allá"; siempre a dimensiones un poco extrañas que, o nos socavan la seguridad en la que estamos plantados, o las condenamos definitivamente a la locura, la arbitrariedad, la vaguedad de quien no sabe razonar. Cualquiera que sea el juicio del lector, nos permitiremos, por lo menos, a modo de conclusiones, los siguientes enunciados, resultantes de las relaciones que hemos querido trazar:

a) A través del relato "El perseguidor" se produce un agenciamiento entre dos dominios diferentes: música y literatura;

b) El relato "EI perseguidor" está completamente atravesado por una "máquina musical-máquina de guerra";

c) El problema del tiempo que se desarrolla en el relato también pasa por la música, o viceversa; y

d) Con "El perseguidor", Julio Cortázar despliega el jazz en su escritura, escribe bebop, expresa bebop en narrativa.

\section{BIBLIO GRAFIA}

Arellano González, Sonia. 1972. "El perseguidor", en: Tres eslabones en la narrativa de Cortázar, Ed. D el Pacífico, Santiago de Chile.

Berendt, Joachim. 1998. El jazz, Ed. Fondo de Cultura Económica, M éxico.

Borello, Rodolfo A. 1980. "C harlie Parker: 'El perseguidor'". En: CuadernosH ispanoamericanos, Ed. Instituto de C ooperación Iberoamericana, M adrid, 0 ct.-D ic., N 0 364-366: 573-594.

Brenet, M ichel. 1962. Diccionario de la música, histórico y técnico, Ed. I beria, Barcelona.

Cifo G onzález, M anuel. 1980. "Relativismo espacio-temporal en 'El perseguidor', de Julio Cortázar". En: Cuadernos H ispanoamericanos, Ed. Instituto de Cooperación I beroamericana, M adrid, 0 ct.-Dic., $\mathrm{N} 0$ 364-366: 414-423.

Cortázar, Julio. 1980. "El perseguidor" y otros relatos, Ed. Bruguera, Barcelona.

D el euze, Gilles y Guattari, Felix. 1997. M il mesetas. Capitalismo y esquizofrenia, Ed. PreTextos, España.

D eleuze, Gilles y Parnet, Claude. 1980. D iálogos, Ed. PreTextos, España.

Estrázulas, Enrique. 1980. "Los perseguidores. Tres acordes para un saxo alto". En: CuadernosH ispanoamericanos, Ed. Instituto de C ooperación I beroamericana, M adrid, $\mathrm{O}$ ctDic., $N$ ㅇ 364-366: 424-430.

Goloboff, M ario. 1998. Julio Cortázar. La biografía, Ed. Seix-Barral, Buenos Aires. Gordon, Samuel. 1980. "Algunos apuntes sobre crítica y 'jazz', en la lectura de 'El perseguidor'". En: Cuadernos H ispanoamericanos, Ed. Instituto de Cooperación Iberoamericana, M adrid, $\mathrm{O}$ ct.-D ic., $\mathrm{N} 0$ 364-366: 595-608.

Soren Triff, Eduardo. 1991. "Improvisación musical y discurso literario en Julio Cortázar". En: Revista I beroamericana, University of Pittsburg, Pittsburg, Penssylvania, U.S.A., Abr.-Sept., № 155-156: 657-663. 